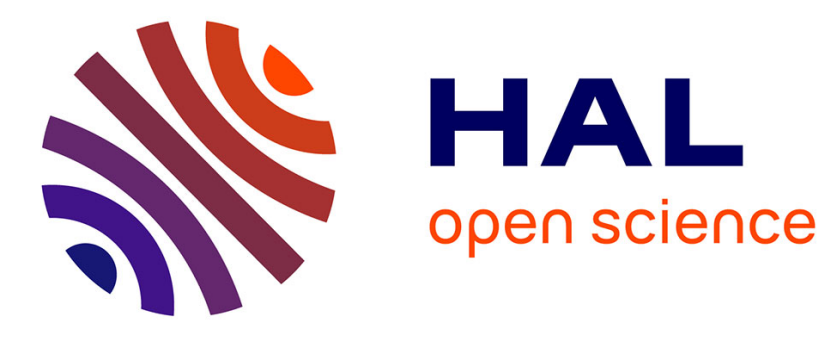

\title{
Shedding light on functional hybrid nanocomposites 19th century paint medium
}

Hélène Pasco, Laurence de Viguerie, Marco Faustini, Cristina Coelho-diogo, Ovidiu Ersen, Christel Gervais, Frédéric Gobeaux, Dris Ihiawakrim, Maguy Jaber, Philippe Walter, et al.

\section{To cite this version:}

Hélène Pasco, Laurence de Viguerie, Marco Faustini, Cristina Coelho-diogo, Ovidiu Ersen, et al.. Shedding light on functional hybrid nanocomposites 19th century paint medium. Advanced Functional Materials, 2022, 32 (4), pp.2106346. 10.1002/adfm.202106346 . cea-03343140

\section{HAL Id: cea-03343140 https://hal-cea.archives-ouvertes.fr/cea-03343140}

Submitted on 2 Nov 2021

HAL is a multi-disciplinary open access archive for the deposit and dissemination of scientific research documents, whether they are published or not. The documents may come from teaching and research institutions in France or abroad, or from public or private research centers.
L'archive ouverte pluridisciplinaire HAL, est destinée au dépôt et à la diffusion de documents scientifiques de niveau recherche, publiés ou non, émanant des établissements d'enseignement et de recherche français ou étrangers, des laboratoires publics ou privés. 


\section{Shedding light on Functional Hybrid Nanocomposites $19^{\text {th }}$ century paint medium}

Hélène Pasco ${ }^{1,2}$, Laurence de Viguerie ${ }^{1}$, Marco Faustini ${ }^{2}$, Cristina Coelho-Diogo ${ }^{3}$, Ovidiu Ersen $^{4}$, Christel Gervais ${ }^{2}$, Frédéric Gobeaux ${ }^{5}$, Dris Ihiawakrim ${ }^{4}$, Maguy Jaber ${ }^{1}$, Philippe Walter $^{1}$, Clément Sanchez ${ }^{2,6}$

${ }^{1}$ Sorbonne Université, CNRS UMR 8220, LAMS, 4 place Jussieu 75252 Paris cedex 05, France

${ }^{2}$ Sorbonne Université, CNRS UMR 7574, Collège de France, LCMCP, 4 place Jussieu 75252 Paris cedex 05, France

${ }^{3}$ Sorbonne-Université, CNRS, Institut des Matériaux de Paris-Centre, FR2482, F-75005, Paris, France

${ }^{4}$ Institut de Physique et Chimie des Matériaux (IPCMS), UMR 7504 CNRS - Université de Strasbourg, 23 rue du Loess, BP43, 67034 Strasbourg Cedex 2, France

${ }^{5}$ Université Paris-Saclay, CEA, CNRS UMR 3685, 91190 Gif-sur-Yvette, France

${ }^{6}$ Chemistry of ultradivided matter, USIAS Chair, Université de Strasbourg Institut of Advanced Studies, 67000, Strasbourg, France

E-mail: laurence.de_viguerie@ @orbonne-universite.fr

Keywords: Hybrid nanocomposites, J. M. W. Turner's practices, lead metallogels, gel Mediums

British $19^{\text {th }}$ century painters formulated gelled systems described as "the most facile and versatile vehicle(s) that any painter has ever had at his disposal". ${ }^{[1]}$ These so-called megilps or gumtions, are based on common paint materials i.e., linseed oil, lead acetate and mastic resin, and allowed painters such as J.M.W Turner to improve their paint's properties and achieve unprecedented effects in an age of innovation.

To propose a structural scheme of these hybrid systems of unprecedented complexity, it was paramount to design and study model systems using a broad combination of advanced spectroscopic techniques and microscopies. Through this approach, we can describe gel Mediums as nanocomposites, based on lead metallogels co-existing with lead oxide nanoparticles and a partially polymerised fraction. These functional hybrid materials created a real breakthrough on $19^{\text {th }}$ century paints performances.

\section{Introduction}


Research on the paint formulations employed by artists is not only a current trend in contemporary conservation science and technical art history, but is something that has preoccupied painters throughout the centuries. ${ }^{[2]}$ Most artists are constantly looking for new functional materials or paint techniques in order to achieve new effects, for their works to last longer, to better express their intentions than before. In the 19th century, technical breakthroughs allowed a major turning point in the artists' way of painting: the use of tube paints mixed with additional mediums allowed them to paint outside, faster and to use brush marks or impasto to give materiality to their paint. British chemists and colormen have developed gel Mediums called "gumtions" or "megilps", a clever mixture of oil, mastic resin and lead, which were widely used by painters and in particular J.W.M. Turner to capture the moment and immortalise their feelings. Many artists, admiring the rich shadows and impastos in the works of Rembrandt and his contemporaries, looked for materials to emulate them ${ }^{[3]}$ and combined bitumen for creating translucent browns with gel Mediums for the transparency and consistency that it gave to paint. The mixture of oil paint and gel exhibits fascinating rheological properties ${ }^{[4]}$ allowing artists to easily tune the paint handling. While acknowledging it, contemporary writers such as R. Redgrave (1804-1888) have blamed its over-use for the dramatic drying issues, in particular severe cracks and disruptions in the paint films, very common at that time. To better understand such behaviour and possible degradation of paint films, it is necessary to investigate the nature and the chemical behaviour of these complex functional materials both on mesoscopic and nanoscopic scales. Previous reconstructions of historical recipes, adding lead acetate to a ternary system based on mastic resin, linseed oil and turpentine, led us to propose different hypotheses on the nature of the gels. $^{[4]}$

The term gel includes a wide-range of chemical systems with various fields of application such as biomedical, ${ }^{[5]}$ food ${ }^{[6]}$ or restoration of works of art. ${ }^{[7]}$ There are many ways to define 
a gel and, as underlined by Weiss \& Terech, ${ }^{[8]}$ all definitions try to connect the micro- and macroscopic properties of gel materials. The main differences are attributed to the distinct driving mechanisms for the gelation process: cross-linking vs. self-organization. Generally, this distinction is referenced as "chemical" or "physical" gels respectively. The former are thus qualified with respect to the covalent nature of the bonds (typically formed by polymerization) and their irreversible nature. These gels are relatively stable at high temperature and at different $\mathrm{pH}$. The latter are conversely sensitive to changes in parameters (temperature, $\mathrm{pH}$, ionic strength, etc.) and therefore reversible. This is due to the weak bonds such as electrostatic interactions, Van der Waals forces, hydrogen bonds etc $^{[9]}$, that structure the material into a supramolecular network. However, this simple picture hides a more complex situation with numerous systems displaying intermediary organization and properties, as coordination based gels that display properties of either physical or chemical gels in different conditions. ${ }^{[10]}$ As an example, metallogels show a relatively high mechanical strength and thermal stability: ${ }^{[11]}$ in these systems, a metallic compound is part of the supramolecular gel as a labile ion interacting with the organic molecules, or incorporated in the Low Molecular Weight Gelator (LMWG) structure, forming a coordination complex. Because of the presence of a metal in their structure, metallogels are extraordinarily versatile. One can also note the numerous topologies, structures and reactivities of metal coordination polymers in which metal carboxylates have emerged as a large family of open framework materials. ${ }^{[12]}$ Lead (II), with its large radius, flexible coordination environment, and variable stereo-chemical activity, has been reported in both metallogels ${ }^{[11,13]}$ and metal coordination polymers. $^{[12]}$

Historical megilps and gumtions are gel Mediums, based on the gelation properties of mixtures of mastic resin with lead compounds in oil. ${ }^{[14]}$ Lead can be introduced as powder of litharge $(\mathrm{PbO})$ during the heating of oil, or as powder or saturated solution of lead acetate $\left(\mathrm{Pb}\left(\mathrm{CH}_{3} \mathrm{COO}\right)_{2}\right)$. Mastic resin, a natural plant exudate harvested from Pistacia lentiscus L. var. 
Chia trees, can be added by dissolution in turpentine, or as a finely ground powder. The spectroscopic study of the mixture between mastic resin and lead revealed to be quite intricate $^{[4]}$ due to the complex nature of the mastic resin, composed of a polymeric fraction (cis-1,4-poly-beta-myrcene) and at least 31 different triterpenic compounds molecules. ${ }^{[15]}$ To precise the origin of the gelation and propose a structural scheme of these paint systems, we designed model systems and investigated them in parallel with reconstructions thanks to spectroscopic methods combined with small angle X-ray scattering (SAXS) and electron microscopies which allow to probe molecular interactions as well as supramolecular organization.

\section{Results and Discussion}

\subsection{Molecular Interactions}

\subsubsection{Design of model systems}

In the field of cultural heritage and artistic paint formulations, a common approach is to reproduce historical recipes to get close to the materials used by painters and study their macroscopic properties. ${ }^{[16]}$ In the case of gel Mediums, based on natural components containing a large number of compounds, no conclusion could be drawn about the gelation process from reconstructions of historical recipes. ${ }^{[4,17]}$ Variations and simplifications were thus explored alongside the reconstructions ${ }^{[18]}$ and a simplified model system was adopted to shed light on the molecular reactions involved in the gelling process, an approach that has proved particularly useful in the case of complex living or metastable systems, such as biological systems for food or drug applications. ${ }^{[19,20]}$

The first step of this study was then to define appropriate model systems, i.e. simplified gel formulations. We have shown before that oil is not necessary to form a gel (the simple combination of dissolved mastic resin with a $\mathrm{Pb}$-compound forms a gel whereas oil with a $\mathrm{Pb}$ compound does not), although the presence of oil certainly leads to the formation of lead 
carboxylates which may participate to the gelation process. As the gels formed without oil exhibit comparable rheological properties and supramolecular lamellar organization as the systems with oil, ${ }^{[4]}$ we decided to use them as a simplified model. Moreover we also investigated the possibility to obtain model gels using one single triterpenoid instead of the mastic resin. ${ }^{[18]}$ Lupeol and oleanolic acid (Figure 1), were both investigated as they are commercially available and known to be present (or with a close structure) in mastic resin: ${ }^{[15]}$ they were dissolved in turpentine prior to addition of lead acetate trihydrate. Only oleanolic acid (minimum ratio: $\mathrm{n}(\mathrm{PbOAc}) / \mathrm{n}($ triterpenoid $) \approx 0.1$ ) led to the formation of a gel, pointing out that the acidic group play a role in the gelation process. We thus decided to investigate model systems composed of oleanolic acid (noted OA) dissolved in turpentine and then ground with lead acetate trihydrate (noted PbOAc) or lead oxide $(\mathrm{PbO})$. All investigated formulations, are summarized in SI (table S1); composition and "ageing" time before measurement.

The model systems exhibit similar rheological properties (Figure S1) as the ones, previously published $^{[4]}$ of reconstructions of historical recipes, with elastic modulus $G^{\prime}$ at least one order of magnitude higher than the viscous modulus G" over a large range of stress (rheological definition of a gel). Systems with oleanolic acid instead of mastic resin exhibits higher moduli and yield stress, indicating a stronger cohesion of the system. 

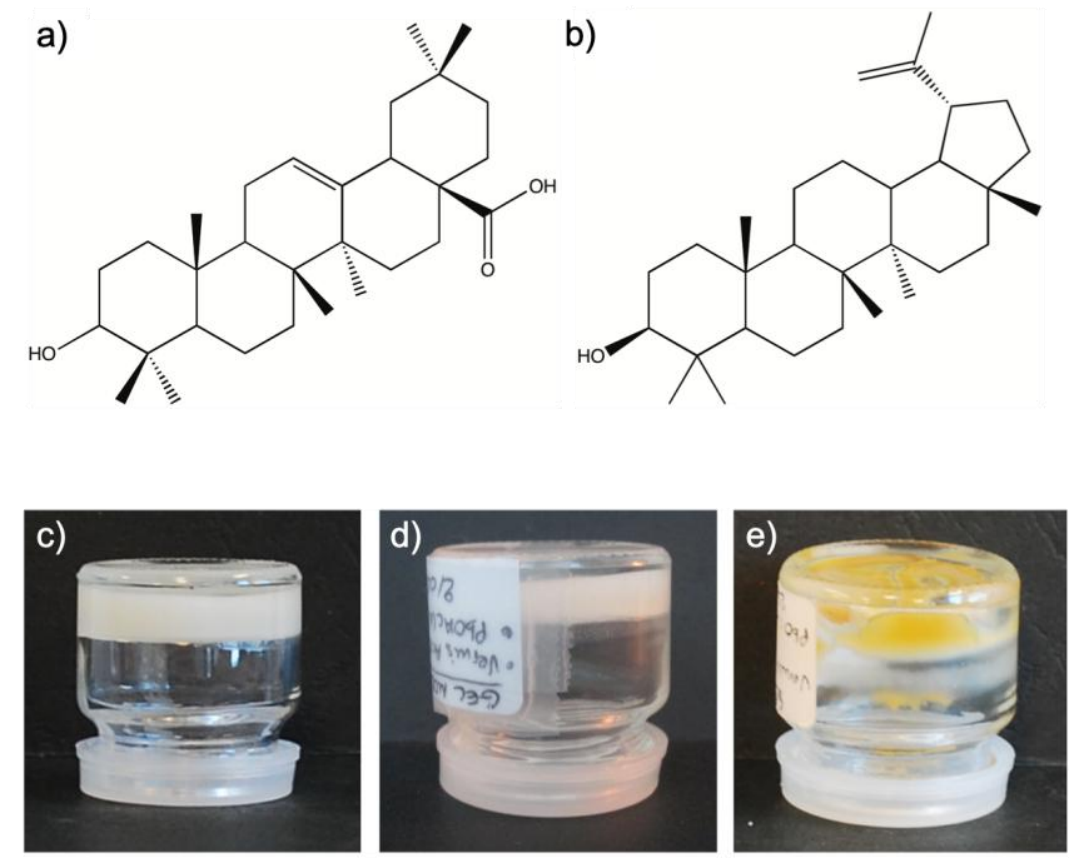

Figure 1: Commercial triterpenoids: oleanolic acid (OA) (a) and lupeol (b). Investigated systems: "mastic resin + turpentine + PbOAc" (c), "OA + turpentine + PbOAc" (d) and "OA + turpentine $+\mathrm{PbO}^{\prime}(\mathrm{e})$.

\subsubsection{Spectroscopic studies}

Multi-reflexion attenuated total reflectance Fourier-transform infrared (ATR FT-IR), solidstate ${ }^{13} \mathrm{C}-\mathrm{NMR}$ and ${ }^{207} \mathrm{~Pb}-\mathrm{NMR}$ spectroscopies were used to characterize the model systems.

The FTIR-ATR spectra of the "OA + turpentine + PbO" system (Figure 2, left) shows a band at $1520 \mathrm{~cm}^{-1}$ that is not present in any of the components of the mixture, whose intensity increases with the quantity of lead compound in the gel. This band has to be ascribed to new carboxylate species, formed by the interaction of lead with the acidic group of the oleanolic acid. It is the second time that such lead-resin carboxylates are mentioned in the literature, being recently identified in paintings (pigments-natural resins systems). ${ }^{[21]}$ This band is relatively broad, likely because of the numerous configurations that can be adopted by lead carboxylates. ${ }^{[22]}$ It is also interesting to note the existence of two smaller bands at 1580 and $1545 \mathrm{~cm}^{-1}$ ("zoom" in Figure 2, right) that could indicate the formation of different resinate species in the gel with different coordination modes. The presence of vibration bands between 1500 and $1600 \mathrm{~cm}^{-1}$ is confirmed in all model systems (as seen in Figure 2, right). In "OA + 
turpentine + PbOAc" systems, a single band is present at $1541 \mathrm{~cm}^{-1}$ which suggests that one coordination mode is prominent here. As this band is rather large and asymmetric, contributions of other coordination modes (in particular at $1520 \mathrm{~cm}^{-1}$ ) are also possible. In "mastic resin + turpentine $+\mathrm{PbOAc} "$ mixture, a band at $1514 \mathrm{~cm}^{-1}$ is present (although less visible than in the "OA + turpentine $+\mathrm{PbO}$ " system), and at high lead acetate ratio, two other ones at 1574 and $1541 \mathrm{~cm}^{-1}$ (Figure 2, right and S2). One can also notice the presence of the mastic resin carbonyl stretching band $\left(c a .1710 \mathrm{~cm}^{-1}\right)$, since the resin contains a lot of carboxylic acid and ketone groups; this band does not undergo noticeable change when the lead compound is added, suggesting that only few carbonyl moieties interact with the lead.
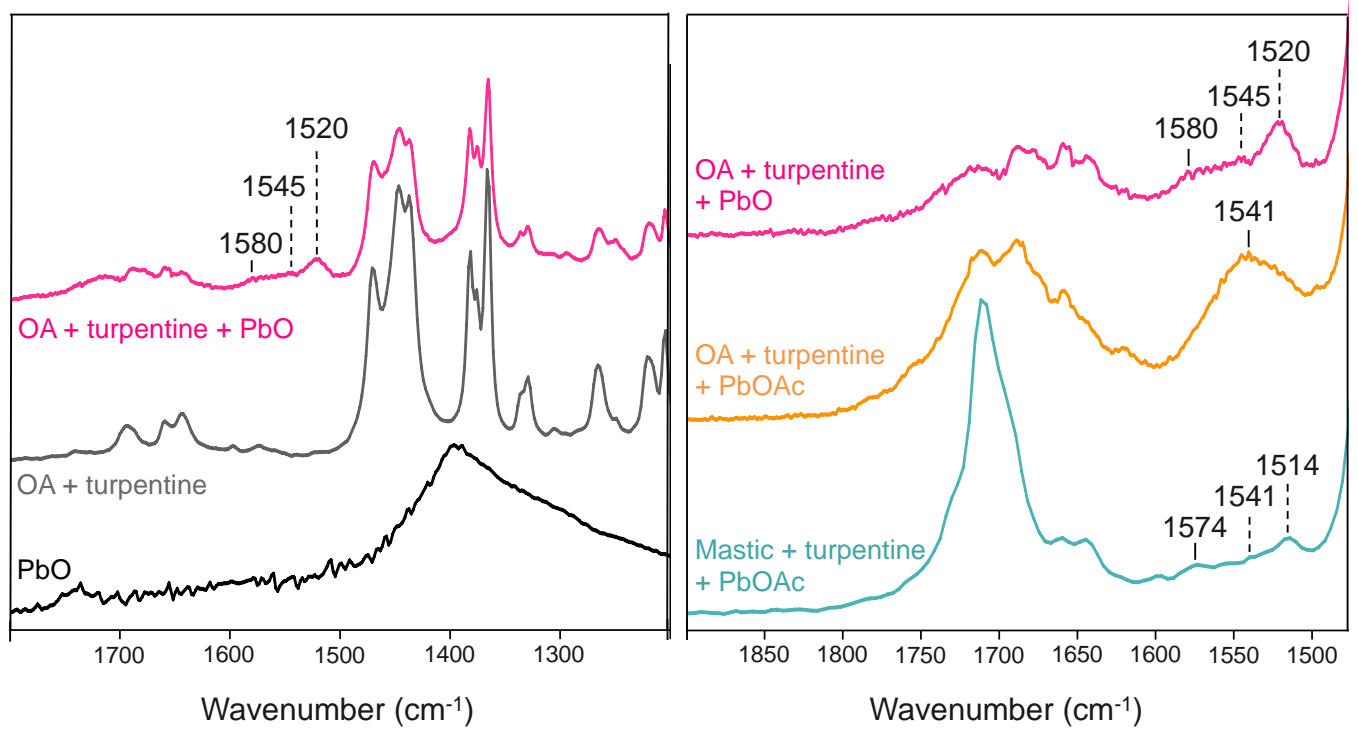

Figure 2: FTIR spectra of: Left: model system "OA + turpentine $+\mathrm{PbO}$ " and individual components; Right: comparison between three model systems (molar ratio between the lead compound and the triterpenic fraction of 2, age of the systems: 2-3 days).

The identification of both symmetric and asymmetric carboxylate bands $\left(v(\mathrm{COO})_{\mathrm{sym}}\right.$ and $\left.v(\mathrm{COO})_{\text {asym }}\right)$ would allow the determination of the different coordination modes: their difference indicates whether the metal is monodentate $(\Delta v \sim 200-300)$, bidentate bridging $(\Delta v \sim 150-180)$ or bidentate $(\Delta v<100) .{ }^{[23]}$ Unfortunately, it is difficult to conclude about the presence of a symmetric band, probably hidden in the multiple bands of the solvent (turpentine) and the resin in the $1300-1450 \mathrm{~cm}^{-1}$ zone (Figure S2). However a shoulder is 
visible at high lead molar ratio (Figure S2) at $1409 \mathrm{~cm}^{-1}$, which is close to values found in the case of lead soaps ${ }^{[24]}$ and may be inferred to the carboxylate symmetric band. Bidentate or bidentate bridging are then the most probable coordination modes, as confirmed in the review made by $\mathrm{Hu}$ et al. $^{[12]}$ showing that carboxylate groups in many of lead (II) coordination polymers form more than a single type of coordination mode and among all of these modes maximum number belong to chelating mode.

${ }^{13} \mathrm{C}$ CP-MAS NMR experiments were also carried out on the different systems at $\mathrm{T}=223 \mathrm{~K}$ $\left(v_{\text {rot }}=10 \mathrm{kHz}\right)$. A new chemical environment appears after mixing the different components, at $c a .180 \mathrm{ppm}$ corresponding to the acidic carbons region. In the spectrum obtained for "OA + turpentine + PbOAc" system, oleanolic acid peaks can be easily identified (Figure S3). We can see that the carboxylic acid carbon is deshielded in the gel i.e., after the addition of lead acetate (186 ppm). This reinforces the previous assumption about the interaction between the lead compound and the acidic group of the resin/oleanolic acid.

To investigate possible complementary reactions, we also monitored the proportion of $\mathrm{C}=\mathrm{C}$ in the gel and the "OA + turpentine" mixture, by integration of the concerned peaks ${ }^{[25]}$ of the ${ }^{13} \mathrm{C}$ RMN spectra: the ratio $\mathrm{C}=\mathrm{C} / \mathrm{C}_{\text {aliphatic }}$ decreases slightly in the gel ( $c a .17 \%$ in the varnish and only $12 \%$ in the gel, calculated from the spectra figure S3).

${ }^{207} \mathrm{~Pb}-\mathrm{NMR}$ experiments were carried out to investigate the local environment of $\mathrm{Pb}$ in the gels and clarify the coordination of $\mathrm{Pb}$ in the complex ( $\mathrm{Pb}$ (II) has a very flexible coordination sphere, since it is known to exhibit a coordination number ranging from 3 to 12$).{ }^{[26]}$

WURST CPMG (see Experimental) static NMR spectra obtained at $243 \mathrm{~K}$ for a gel made of oleanolic acid and PbOAc (molar ratio 18 and 6) indicate similar CSA (Chemical Shift Anistropy) parameters as for PbOAc (Figure S4 and Table S2). In this configuration, we could not obtain spectra with sufficient signal-to-noise ratio with a lower amount of PbOAc. 
Hence, we carried out CP experiments at lower temperature $(100 \mathrm{~K})$ and with higher magnetic field (i.e., 800 vs. $700 \mathrm{MHz}$ ). These conditions allowed the analysis of a gel with stoichiometric proportions between the triterpenoid and the lead, so as to avoid detecting only excess lead acetate trihydrate. The ${ }^{207} \mathrm{~Pb}-\mathrm{NMR}$ spectrum of the gel prepared with equimolar proportions exhibits slightly different NMR parameters but the line shape is again very similar to the lead precursor (Figure S5 and Table S2) confirming unchanged coordination upon reaction: $\mathrm{Pb}$ bound with carboxylic acid groups of the resin has a probable coordination of 8 or 9 as well as $\mathrm{Pb}$ present in the excess of lead acetate (as described in the existing literature $\left.{ }^{[27-29]}\right)$. As proposed for yttrium acetate tetrahydrate ${ }^{[30]}$, bidentate bridging, also called "polymeric" coordination mode seems the most probable in this case: it allows high $\mathrm{Pb}$ coordination (8 or 9) without too much geometrical constraints and supramolecular selfassembly of the complexes. Karl-Fischer measurements indicate that $c a$. 1 to $2 \mathrm{~mol}_{2} \mathrm{O}$ are found for $1 \mathrm{~mole} \mathrm{~Pb}$; these water molecules are probably able to complete the coordination up to 8 (Figure 3). FTIR-ATR spectra also suggest the coexistence of several coordination modes, with transition processes depending on the ratio Pb-triterpenoids (as in metallogels lithocholate- $\mathrm{Cu}^{2+}$ described in ${ }^{[11]}$. 


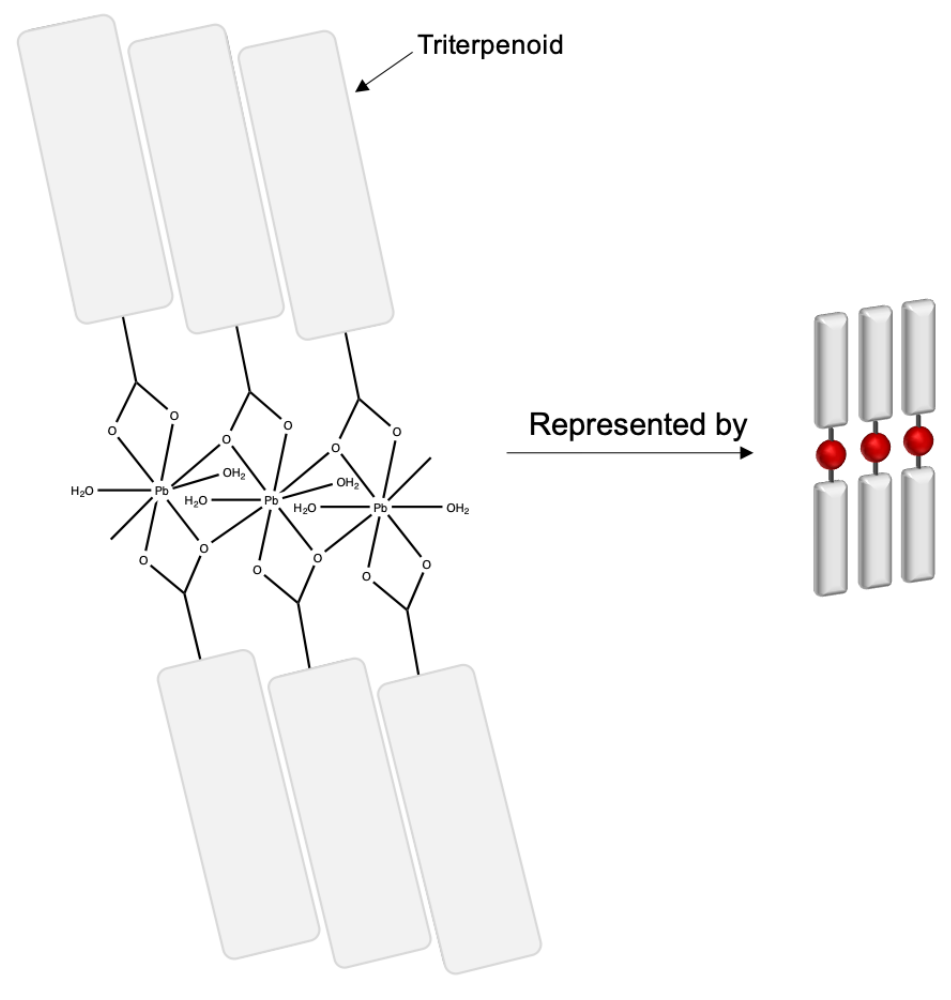

Figure 3: Schematic representation of the self-assembly of the oleanolic acid $(\mathrm{OA})-\mathrm{Pb}^{2+}$ complex under bidentate bridging mode.

\subsection{Supramolecular organization}

Regarding the structural characterization of the gels, two complementary techniques were used to probe locally and globally the supramolecular organization. Figure 4a displays the evolution of the SAXS-WAXS profile during the formation of a gel "mastic resin + turpentine + PbOAc" during 20 hours (at low molar ratio to slow down the gelation rate that is much increased in capillaries). As the scattering intensity increases over time, we can clearly observe the appearance of a $q^{-2}$ slope in the 0.03-0.1 $\AA^{-1} q$-range, typical for 2-dimensional objects such as platelets or lamellar compounds (detail in Figure S6) followed by a correlation peak, at $q$ ca. $0.26 \AA^{-1}$ corresponding to a characteristic distance of $c a .2 .4 \mathrm{~nm}$. When the concentration of metal ions is varied (Figure S7), the curves are close to the one at molar ratio 0.16 , and the position of the correlation peak is almost unchanged, indicating that the amounts of metal ions exhibit very little effect on the arrangement of triterpenoid species. 

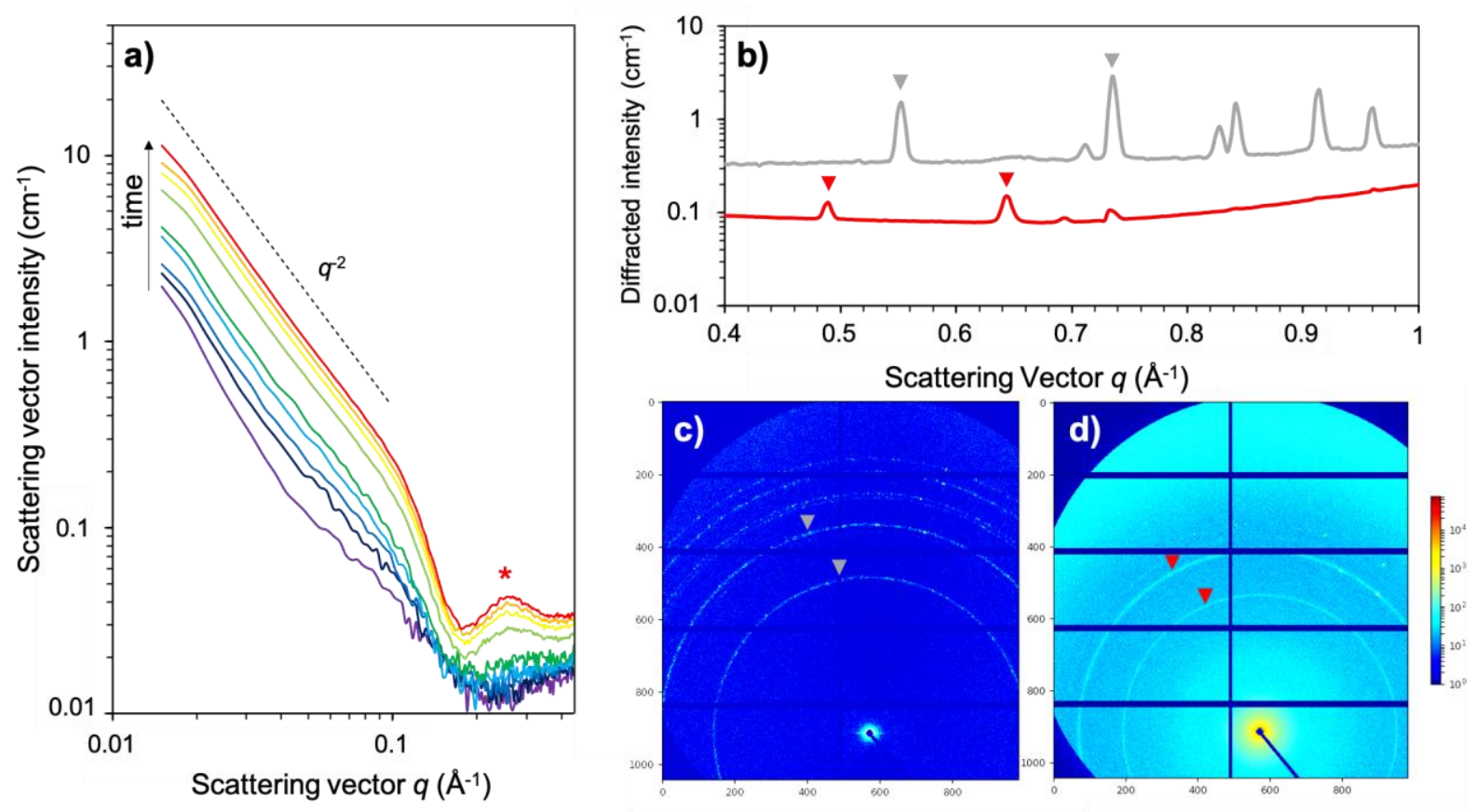

Figure 4: a) SAXS-WAXS profiles of the monitoring of the gel formation ("mastic resin + turpentine + PbOAc", molar ratio 0.2) during 20 hours (raw curves subtracted by the linear fit of the varnish curve); b) WAXS profile of: (red) same gel (middle of the capillary) and (grey) PbOAc powder in turpentine (bottom of the capillary, unsolubilized). The red arrows indicate the characteristic reflections of the lead specie in the gel, while the grey arrows correspond to the $\mathrm{PbOAc}$ salt; $\mathrm{c}$ ) and d) corresponding 2D patterns.

In the $q$-range from 0.5 to $1 \AA^{-1}$, the WAXS profiles (Figure $4 \mathrm{~b}$ ) display the reflection pattern of the crystalline compounds. The gel was compared to a simple mixture of turpentine and PbOAc in which no reaction occurs. We can clearly see the PbOAc diffraction peaks in the "turpentine + PbOAc" mixture, whereas in the gel two different peaks are clearly visible at $c a$. 0.49 and $0.65 \AA^{-1}$, corresponding to characteristic distances of 1.28 and $0.96 \mathrm{~nm}$ which can be linked respectively to the length of pentacyclic triterpenoids, such as OA (ca. 1.2-1.3 nm according to the literature $)^{[31]}$ and to the width of the OA molecule $(c a \cdot 0.45-0.5 \mathrm{~nm}$, according to Avogadro software), which would roughly represent the distance between two lead atoms in the complex (Figure 3). As seen in Figure S8, displaying the evolution of the 0.5 to $1 \AA^{-1}$ region during the kinetics study, these two peaks increase during the gel formation. One has also to underline the corresponding 2D patterns (Figure $4 \mathrm{c}$ and $\mathrm{d}$ ). The reflections of lead acetate (grey arrows) are different from those of lead in gel (red arrows): in 
the first case, the diffraction is discontinuous and the intensity markedly varies, suggesting the presence of large crystallites. Conversely, we observe smooth uniform patterns in the gel indicating randomly oriented objects.

SAXS-WAXS was combined with cryo-microscopy (cryo-Scanning Transmission Electron Microscopy STEM and -TEM) to confirm these observations and investigate local inhomogeneities. In contrast to SAXS and Freeze Fracture Electron Microscopy (FFEM presented in de Viguerie et al. $)^{[4]}$, cryo-STEM and -TEM micrographs revealed a high level of complexity in these systems, which exhibit organized structures co-existing with dispersed nanoparticles. For both analysed molar ratio (0.1 and 0.5$)$, the samples are quite heterogeneous and consist in two types of structural elements:

i) sheet-like arrangements made of few lamellas, very probably dispersed in a continuous phase, with interlamellar distances between 1 and $2.5 \mathrm{~nm}$ (Figure 5) depending on the solvent/molecules penetration, in good agreement with the characteristics distances measured by SAXS-WAXS; note that the smallest distances are measured for the longest $2 \mathrm{D}$ arrangements which seem to be characterized by a very high long range order, as it can be observed in Figure 5;

ii) nanoparticles of different sizes (Figure 6 and S9), characterized by a bimodal distribution in the analysed areas (Figure S9a-c), which are either dispersed or aggregated (both indicated by arrows in Figure 6a and b) for forming larger 2D objects (Figure 6c and d, and S9d). It is important to underline that both types of structures coexist in the analysed specimens and also that some sheet-like arrangements seem to be constituted of well-arranged nanoparticles (Figure 6).

From the crystallinity point of view, structural analyses were carried out by cryo-TEM at different times after the gel preparation (the systems being kept in sealed vials); they revealed a considerable ageing effect in the gel, although the spectroscopic measurements did not show 
any evolution over the same period (except for the evaporation of the solvent), nor did the SAXS measurements (carried out in sealed capillaries 3 months after gelation). In particular, few days after the gel preparation amorphous nanoparticles are mainly visible, whereas smaller crystallites appear after few months. Electron diffraction combined with the analysis of the high resolution TEM images allowed us to evidence the presence of both lead acetate and lead oxide phases in the nanoparticles (Figure S10 and S11), probably due to the reaction process with lead and the presence of residual water in the system; lead oxide phases $(\mathrm{PbO}$ and $\mathrm{PbO}_{2}$ ) being observed only after a certain ageing time.
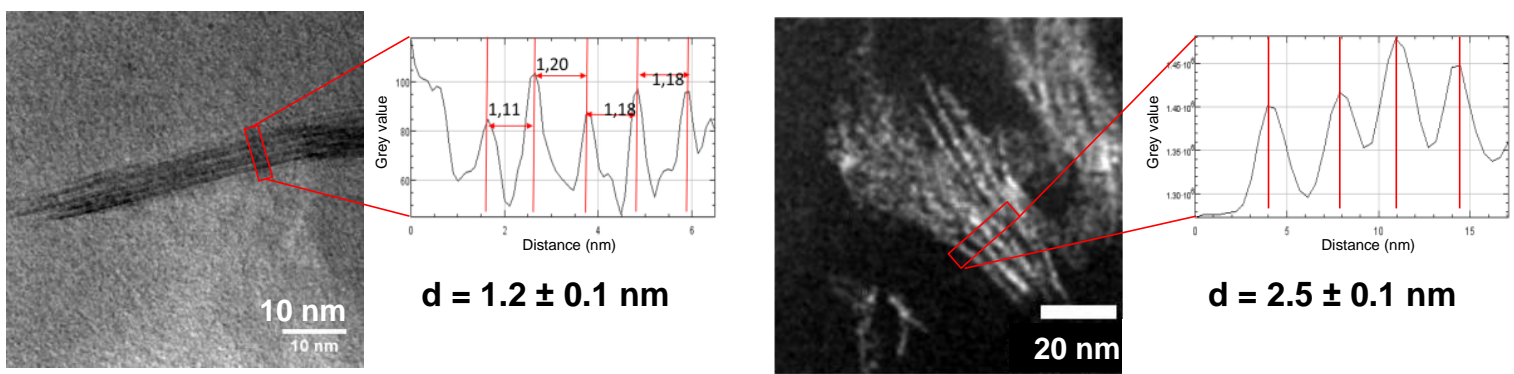

Figure 5: Typical cryo-TEM (left) and Cryo-STEM (right) images of a gel (made of «OA + turpentine $+\mathrm{PbOAc} »$, molar ratio 0.1 - age of the systems: 4 months) and the quantification of the interlamellar distances measured between lamellas. 

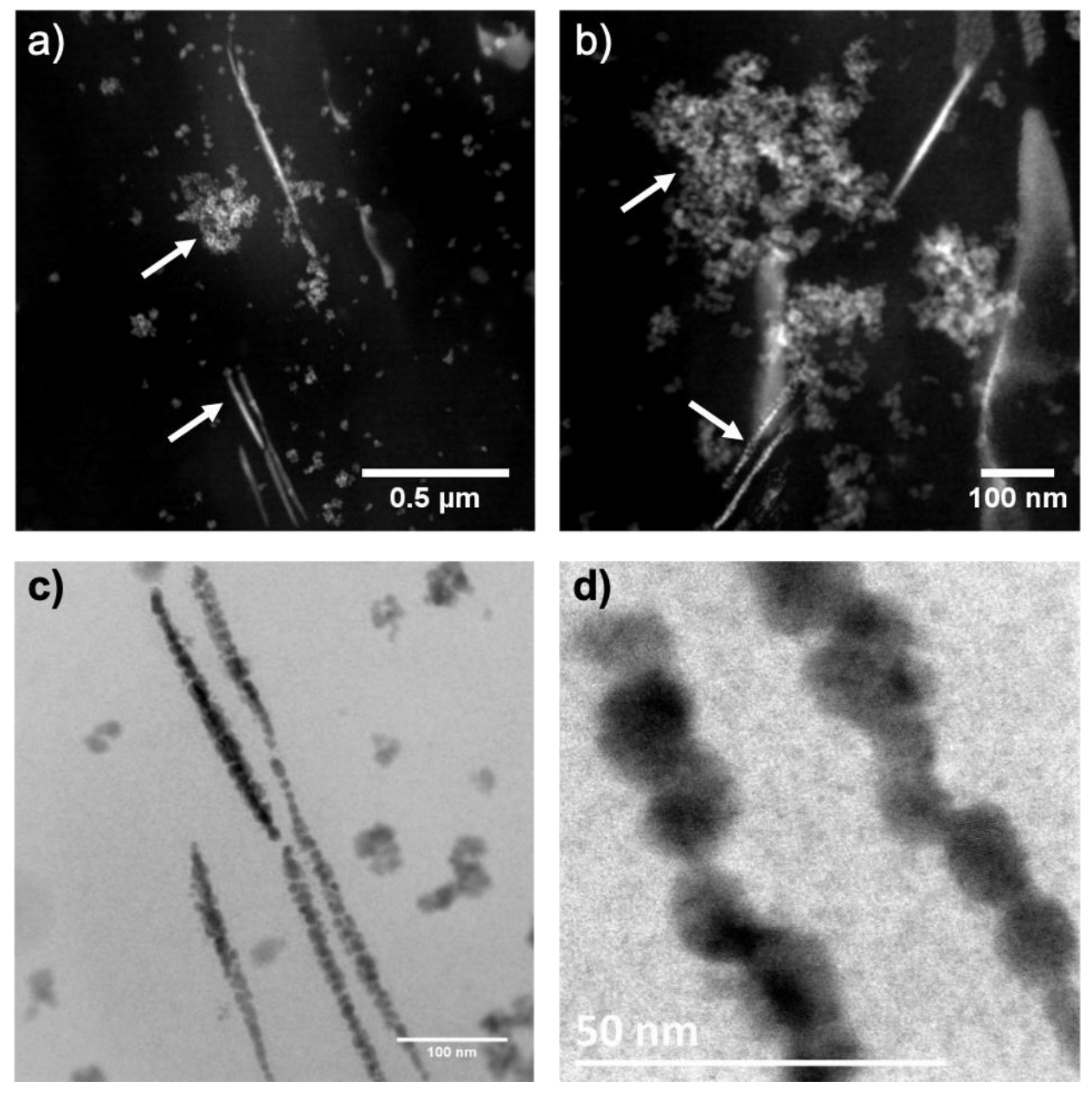

Figure 6: a) and b) Typical cryo-STEM images highlighting the coexistence of the two types of arrangements, sheet-like and nanoparticle-based, c) and d) Cryo-TEM images showing the make-up of some lamellar structures from the 2D arrangement of individual nanoparticles. Gel made of « mastic + turpentine + PbOAc », molar ratio 0.5 - age of the systems: 4 months.

\subsection{Discussion: towards a complete picture of these hybrid functional materials}

On the basis of the numerous experimental data reported in this work, we can propose the following simplified formation process of these hybrid nanocomposite systems:

Micron-sized hydrated lead acetate precursors, carboxylated triterpenes, solvent (turpentine i.e., mainly monoterpenes) and poly-beta-myrcene oligomers are mixed manually and mechanically. FTIR-ATR and ${ }^{13} \mathrm{C}-\mathrm{NMR}$ demonstrated the interaction of the lead compound and the carboxylic acid moieties of the triterpenoids, by the formation of resinate bands in FTIR (1500-1600 $\left.\mathrm{cm}^{-1}\right)$ and of a new chemical environment in the acidic carbon region in NMR ( $c a .180$ ppm). The coordination between lead cations and carboxylic acid functions of 
the triterpenoids drives the dissolution of the lead precursors in the organic mixture, and the subsequent formation of lamellar triterpenoids metallogels such as the more studied sterol metallogels based on lithocholates in presence of $\mathrm{Pb}$ or $\mathrm{Cu}$ ions, ${ }^{[11]}$ that display continuous lamellar structures. As shown by NMR, the lead (II) retains a high coordination $\left(\mathrm{Z}=8,{ }^{207} \mathrm{~Pb}\right.$ NMR) satisfied by chelating-bridging carboxylate ligands and water molecules, whose presence was confirmed by Karl-Fischer measurements. This process leads to a decrease in the size of the initial lead acetate particles, which gradually become nanometric (as seen thanks to cryo-TEM) and therefore more reactive as their surface-to-volume ratio increases.

As we are in the presence of residual water (still according to Karl-Fischer measurement), some of these nanoparticles can begin to hydrolyse to form, via hydrolysis-condensation reactions, other nanoparticles based on lead hydroxide and oxides $\left(\mathrm{PbO}, \mathrm{PbO}_{2}\right)$, observed in cryo-TEM micrographs. It can be stressed that, as lead oxide particles are semiconductors (band gap of 2.6-2.7 eV) and therefore photocatalysts, they may create holes and precursor electrons of radical species in the presence of visible light. It is also quite possible that these radicals (observed in previous EPR experiments ${ }^{[4]}$ ) contribute to the polymerisation processes of certain double bonds present in the varnish components and observed by ${ }^{13} \mathrm{C}-\mathrm{NMR}$.

Based on this description, a simplified representation of these complex materials, which are actually hybrid nanocomposites containing class I and class II hybrids according to the international classification ${ }^{[32,33]}$ i.e., with both strongly and weakly bonded organo-mineral interfaces, is shown in the diagram in Figure 7. This cartoon shows domains consisting of lamellar metallogels (in red) based on lead carboxylates functionalized by terpenes, which are more or less swollen by solvent molecules or unreacted triterpenes, residual lead acetate nanoparticles (blue), nanoparticles based on lead oxide or hydroxide (green). Partially polymerized gel can be also present, possibly in separated domains (as represented here in light orange) in large areas of physical gel (light grey background). 


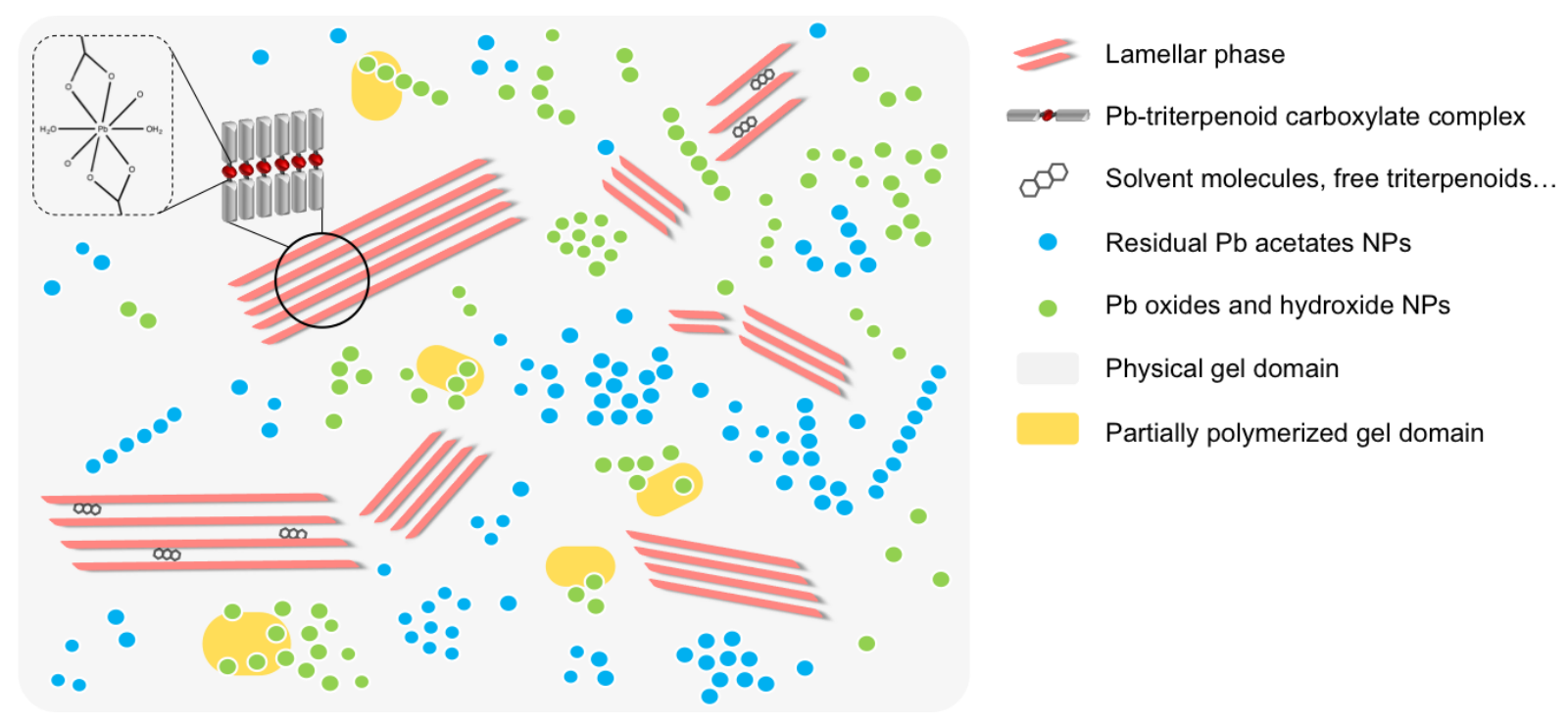

Figure 7: Schematic representation of the system complex functional hybrid materials.

It should be recalled here that this rather complex structural scheme corresponds only to the simplified system and should be modified by taking into account firstly the presence of oil and its interaction with the other components (and in particular lead) and secondly by considering the "real system" used by painters, i.e. the mixing of the gels with the paint (oil and pigments), forming a new functional hybrid material with additional levels of complexity.

\section{Conclusion}

Thanks to the combination of numerous advanced analytical techniques, spectroscopies and cryo-microscopies, $19^{\text {th }}$ century Medium gels that provide paint with attractive handling properties as well as fast drying and increased transparency, can be now described as functional hybrid nanocomposites. If a lead metallogel formation seems to be the driving gelation mechanism, lead acetate and oxide based nanoparticles, and probably partially polymerized organic domains, are also present in this complex hybrid system.

This study opens perspectives in various fields dealing with complex natural systems such as in foods, cosmetics, decontamination, bio-technology, or drugs and pharmaceuticals (where oleanolic acid is used for its healing activity). It also deepens the knowledge in the rising field 
of supramolecular gels and metallogels where understanding the mechanisms of gelation at the molecular level is essential to define strategies for the rational design of new gel systems. ${ }^{[34]}$

Further investigation is now required about the effect of oil in our Medium gels models: the presence in these materials of lead oxide-based nanoparticles with semiconducting properties surely affects their reactivity with oil and, later on, with paint on the artist's palette. This study raises the important issue of how these "living hybrid systems" age in the paint system. Although very attractive from the handling, drying and optical properties point of view, strong degradation have already been observed in $19^{\text {th }}$ century paintings and linked to the use of these peculiar systems.

\section{Experimental Section/Methods}

\section{Materials}

For the recipes of the historical gels and the reconstructions, please report to [4] where the protocols have been adapted from [14]. The sugar of lead referred to in the historical recipes was interpreted as lead acetate trihydrate $\mathrm{Pb}\left(\mathrm{CH}_{3} \mathrm{COO}\right)_{2} \cdot 3 \mathrm{H}_{2} \mathrm{O}_{(\mathrm{s})}$ (Sigma Aldrich, 99\%) to be in the same conditions as the artists. Lead oxide, also purchased from the same supplier, is a mixture of $\alpha$ - and $\beta$-PbO (99+\%). We also employed two different mastic resins (Laverdure 2015, and Kremer Pigmente 2016) both originally harvested from the island of Chios. To make the varnish, we used rectified spirit of turpentine from Sennelier.

Oleanolic acid (> 97\%) and lupeol, as model compounds of the mastic resin, were purchased from Sigma Aldrich. Gels were obtained by mixing $1 \mathrm{~g}$ of turpentine with $50 \mathrm{mg}$ of the triterpenoid. Then different amounts of lead compound were added and grinded into the mixture so as to obtain a molar ratio ranging from 0.1 to 18 (4.5 to $750 \mathrm{mg}$ for PbOAc, 2.5 to $440 \mathrm{mg}$ for $\mathrm{PbO}$ ). Gels obtained were stored in a sealed glass jar. 
All investigated formulations are listed in Table S1. Spectroscopic measurements and SAXS were carried out from few hours to few days after the preparation process. Due to experimental constraints, cryo-microscopies were carried out from few weeks to few months (12 months being the maximum).

FTIR

The analyses were carried out on a Cary 630 device equipped with a Cary 5 bounce Zinc Selenide ATR accessory (from Agilent). Liquid (varnishes) and soft samples (gels) were evenly spread on the large ZnSe crystal, which grants a longer pathlength for the infrared beam, making multi-bounce possible, thus enhancing the detection limit. The spectra were acquired with Microlab software with the following parameters: $650-4000 \mathrm{~cm}^{-1}, 32$ scans, spectral resolution $4 \mathrm{~cm}^{-1}$.

${ }^{13} \mathrm{C}-\mathrm{NMR}$ and ${ }^{207} \mathrm{~Pb}-\mathrm{NMR}$

${ }^{13} \mathrm{C}-\mathrm{NMR}$ experiments were carried out on a Bruker Advance III $500 \mathrm{SB}$ spectrometer (magnetic field: $11.6 \mathrm{~T}$ ). The experiments were conducted at $223 \mathrm{~K}$ at $10 \mathrm{kHz}$ with a $4 \mathrm{~mm}$ rotor. For ${ }^{207} \mathrm{~Pb}-\mathrm{NMR}$ analyses, two setups were used. The first series of experiments were operated on a Bruker Advance III 700 SB spectrometer (magnetic field: 16.3 T), tuned at 146.471 MHz for ${ }^{207} \mathrm{~Pb}$ resonance. The WURST-CPMG (Wideband Uniform rate Smooth Truncation pulses coupled with Carr-Purcell-Meiboom-Gill sequence experiments $)^{[35]}$ were carried out at $243 \mathrm{~K}$ with 2.5 and $4 \mathrm{~mm}$ rotors respectively. ${ }^{1} \mathrm{H} \rightarrow{ }^{207} \mathrm{~Pb} \mathrm{CP}$ experiments were conducted on a Bruker Advance III 800 SB spectrometer (magnetic field: $18.6 \mathrm{~T}$ ) at $100 \mathrm{~K}$ with a $3.2 \mathrm{~mm}$ rotor.

NMR spectra were processed using Topspin software and the line shapes and CSA parameters were simulated using Dmfit program. ${ }^{[36]}{ }^{207} \mathrm{~Pb}$ chemical shifts were referenced using $\mathrm{Pb}\left(\mathrm{NO}_{3}\right)_{2}$ $\left(\delta_{\text {iso }}=-3494 \mathrm{ppm}\right.$ at room temperature $)$.

\section{SAXS}


SAXS measurements were acquired on a laboratory device (LIONS-NIMBE CEA, Saclay, France) at $1.542 \AA$ (Cu source) under vacuum with a Xeuss 2.0 apparatus (Xenocs). The displayed $q$-range from $2.10^{-2}$ to $1 \AA^{-1}$ was attained with a single sample-to-detector distance of $54 \mathrm{~cm}$. The latter was calibrated with tetradecanol and the detector count was normalized by direct beam measurements. The samples were inserted in quartz capillaries (diameter $1 \mathrm{~mm}$, wall thickness $0.1 \mathrm{~mm}$, WJM-Glas) before gelling. Acquisition times were of $45 \mathrm{~min}$, except for the first two hours of the kinetics where it was set to 5 min to monitor the sample evolution with an adequate time-resolution. The treatment of the $2 \mathrm{D}$ images and of the resulting scattering profiles was performed using pySAXS. Standard procedures were applied to subtract background scattering and to normalize the intensities. ${ }^{[37]}$ Varnish (resin + turpentine) profiles could be linearly fitted by a power law $I=0.0302 \times q^{-0.813}$ in the $[0.015$ $\left.0.34 \AA^{-1}\right] q$-range with a 0.9989 correlation coefficient. When it was not possible to directly subtract the experimental varnish profiles, we used that fit.

Cryo-STEM HAADF

For the cryo-TEM and STEM analyses, the gels were prepared using the Tokuyasu method ${ }^{[38]}$ in which the samples are frozen in the presence of sucrose, for improving the cutting procedure, and then sectioned in $80 \mathrm{~nm}$ thick cuts. The ultramicrotomy cuts were stored in liquid nitrogen and transferred in a Cs corrected JEOL $2100 \mathrm{~F}$ microscope operating at $200 \mathrm{kV}$. With the specimen in the cryo-TEM holder allowing to maintain it at low temperature, classical and high resolution TEM analyses were performed in the traditional TEM mode as well as in scanning TEM (STEM) using the Bright Field (BF) and High Angle Annular Dark Field (HAADF) modes.

\section{Supporting Information}

Supporting Information is available from the Wiley Online Library or from the author.

\section{Acknowledgements}


N. Birlirakis and D. Carnevale are gratefully acknowledged for their technical support recording 18.6 T NMR spectra, as well as B. Rigaud for recording ${ }^{13} \mathrm{C}$ NMR spectra. We also express gratitude to L. Laporte for help in recording diffraction and SAXS data on gel systems, and to L. Carlyle for many fruitful discussions on the topic.

This research was supported by the IR-RMN-THC Fr3050 CNRS for recording 18.6 T NMR spectra as well as the French Region Ile de France - SESAME program (700 MHz NMR spectrometer). The PhD of H. Pasco was founded by the ED397 and the LabEx MiChem, part of the French state funds managed by the ANR within the investissements d'avenir program under reference ANR-11-10EX-0004-02.

Received: ((will be filled in by the editorial staff))

Revised: ((will be filled in by the editorial staff)) Published online: ((will be filled in by the editorial staff))

\section{References}

[1] J. Maroger, A la recherche des secrets des grands peintres, Dessain et Tolra, 1986.

[2] Ph. Walter, L. de Viguerie, Nat. Mater. 2018, 17, 106-109.

[3] R. Redgrave, S. Redgrave, A Century of Painters of The English School; with Critical Notices of Their Works, and an Account of the Progress of Art in England, Smith, Elder and Co, London, England 1866, vol. 2, p591.

[4] L. de Viguerie, M. Jaber, H. Pasco, J. Lalevée, F. Morlet-Savary, G. Ducouret, B. Rigaud, T. Pouget, C. Sanchez, P. Walter, Angew. Chem. Int. Ed. 2017, 56, 1619-1623.

[5] N. N. Ferreira, L. M. B. Ferreira, V. M. O. Cardoso, F. I. Boni, A. L. R. Souza, M. P. D. Gremião, Eur. Polym. J. 2018, 99, 117-133.

[6] S. Banerjee, S. Bhattacharya, Crit. Rev. Food Sci. Nutr. 2012, 52, 334-346.

[7] D. Chelazzi, E. Fratini, R. Giorgi, R. Mastrangelo, M. Rossi, P. Baglioni, in Gels and other soft amorphous solids, (Eds: F. Horkay, J. F. Douglas, E. Del Gado), ACS Symposium Series, 2018, vol. 1296, Ch. 15, p 291-314.

[8] R.G. Weiss, P. Terech, Molecular Gels: Materials with Self-Assembled Fibrillar Networks, Springer, Dordrecht, Netherlands 2006.

[9] P. Terech, I. Furman, R. G. Weiss, H. Bouas-Laurent, J. P. Desvergne, R. Ramasseul, Faraday Discuss. 1995, 101, 345-358.

[10] W. Zhang, Z. Wang, L. Tao, K. Duan, H. Wang, J. Zhang, X. Pan, Z. Huo, J. Solid State Electr. 2019, 23, 1563-1570.

[11] H. Wang, S. Song, J. Hao, A. Song, Chem. Eur. J. 2015, 21, 12194-12201.

[12] M.-L. Hu, A. Morsalib, L. Aboutorabi, Coord. Chem. Rev. 2011, 255, 2821-2859.

[13] J. V. Knichal, W. J. Gee, A. D. Burrows, P. R. Raithby, C. C. Wilson, Cryst Eng Comm 2015, 17, 8139-8145.

[14] L. A. Carlyle, The artist's assistant: oil painting instruction manuals and handbooks in Britain 1800-1900, with reference to selected eighteenth-century sources, Achetype Publications, London, United Kingdom 2001.

[15] A. N. Assimopoulou, V. P. Papageorgiou, Biomed. Chromatogr. 2005, 19, 285 - 311.

[16] L. Carlyle, De Mayerne Programme HART Report 2002-2005, FOM-AMOLF, ICN, and 
CCI, 2005.

[17] J. H. Townsend in Historical Painting Techniques, Materials, and Studio Practice (Eds.: A.Wallert, E. Hermens, M. Peek), The J. Paul Getty Trust, United States, 1995, p 176 - 185.

[18] H. Pasco, PhD Thesis, Sorbonne Université (Paris), Nov. 2019.

[19] W.J. Harper, in Milk proteins, from expression to food (Eds: H. Singh, M. Boland, A. Thompson), Elsevier Academic Press, 2009, Ch. 15 (451-467).

[20] J.S. Nowick, Org. Biomol. Chem. 2006, 4, 3869-3885.

[21] T. Poli, A. Piccirillo, M. Nervo, O. Chiantore, in Metal Soaps in Art: Conservation and Research, (Eds: F. Casadio, K. Keune, P. Noble, A. van Loon, E. Hendriks, S. A. Centeno, G. Osmond), Springer International Publishing, 2019, Ch. 8.

[22] F. Casadio, K. Keune, P. Noble, A. van Loon, E. Hendriks, S. A. Centeno, G. Osmond, Eds., Metal Soaps in Art: Conservation and Research, Springer International Publishing, 2019.

[23] K. Nakamoto, Infrared and Raman Spectra of Inorganic and Coordination Compounds, Wiley, New York, USA 1986.

[24] L. de Viguerie, P.A. Payard, E. Portero, Ph. Walter, M. Cotte, Prog. Org. Coat. 2016, 93, 46-60.

[25] M. M. Alam, H. Peng, K.S. Jack, D. J. T. Hill, A. K. Whittaker, J. Polym Sci., part A 2017, 55, 919-927.

[26] M. Vijayan, M. A. Viswamitra, Acta Cryst. 1966, 21, 522-532.

[27] P. G. Harrison, A. T. Steel, J. Organomet. Chem. 1982, 239, 105-113.

[28] R. K. Rajaram, J. K. M. Rao, Z. Kristallogr. Cryst. Mater. 1982, 160, 225-233.

[29] R. G. Bryant, V. P. Chacko, M. C. Etter, Inorg. Chem. 1984, 23, 3580-3584.

[30] F. Ribot, P. Toledano, C. Sanchez, Inorg. Chim. Acta 1995, 185, 239-245.

[31] B.G. Bag, C. Garai, R. Majumdar, M. Laguerre, Struct Chem 2012, 23, 393-398.

[32] M. Faustini, L. Nicole, E. Ruiz-Hitzky, C. Sanchez, Adv. Funct. Mater. 2018, 28 (27), 1704158.

[33] C. Sanchez, F. Ribot, New J Chem 1994, 18, 1007.

[34] P. Dastidar, Chem. Soc. Rev. 2008, 37, 2699.

[35] L. A. O’Dell, R. W. Schurko, Chem. Phys. Lett. 2008, 464(1), 97-102.

[36] D. Massiot, F. Fayon, M. Capron, I. King, S. Le Calvé, B. Alonso, J.-O. Durand, B. Bujoli, Z. Gan, G. Hoatson, Magn. Reson. Chemistry 2002, 40, 70-76.

[37] P. Linder, in Neutrons, X-rays and light: scattering methods applied to soft condensed matter. (Eds: P. Linder, T. Zemb), Delta Series-Elsevier, Netherlands 2002, Ch. 2.

[38] K. Tokuyasu, J. Cell Biol. 1973, 57, 551-565. 
In-depth analytical study on a famous recipe of gel paint media used by $19^{\text {th }}$ century painters reveals:

- their complex driving gelation mechanism: the supramolecular self-assembly of metallogels based on the formation of lead-resin "soaps",

- their nanocomposite nature with the unexpected presence of semi-conductor lead oxide nanoparticles, co-exisiting with lamellar metallogels and partially polymerized gel.

Hélène Pasco ${ }^{1,2}$, Laurence de Viguerie ${ }^{1 *}$, Marco Faustini ${ }^{2}$, Cristina Coelho-Diogo ${ }^{3}$, Ovidiu Ersen $^{4}$, Christel Gervais ${ }^{2}$, Frédéric Gobeaux ${ }^{5}$, Dris Ihiawakrim ${ }^{4}$, Maguy Jaber ${ }^{1}$, Philippe Walter $^{1}$, Clément Sanchez ${ }^{2,6}$

\section{Shedding light on Functional Hybrid Nanocomposites $19^{\text {th }}$ century paint medium}

ToC figure

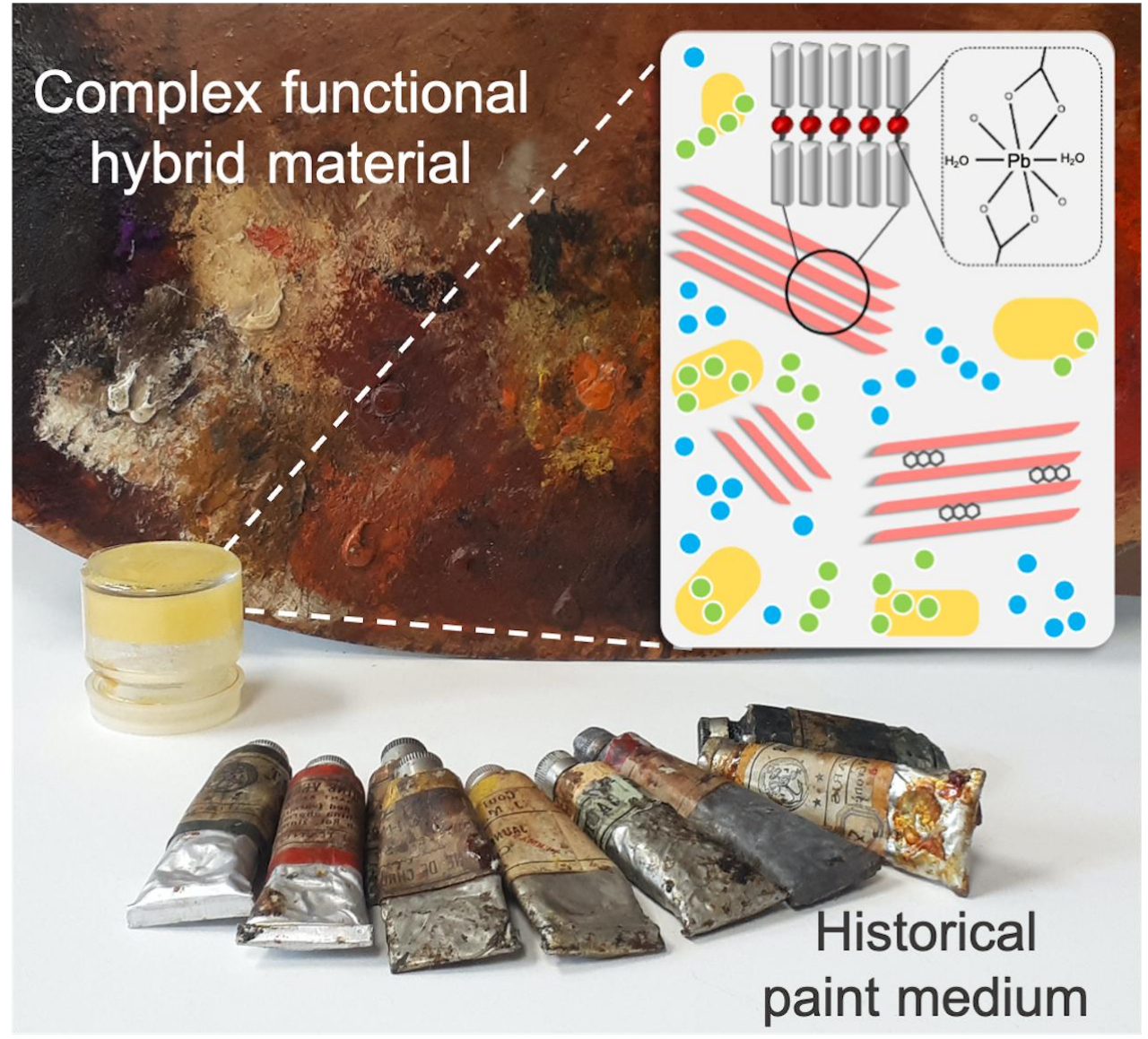

